\title{
Magnitude of Cryptococcal Antigenemia among HIV Infected Patients at a Referral Hospital, Northwest Ethiopia
}

\author{
Awoke Derbie ${ }^{1,2 *}$, Workneh Ayalew ${ }^{3}$, Daniel Mekonnen ${ }^{1,4}$, Megbaru \\ Alemu $^{1}$, Yihun Mulugeta ${ }^{5}$
}

\section{OPEN ACCESS}

Citation: Awoke Derbie, Workineh Ayalew, Daniel Mekonne, Megbaru Alemu, Yihun Mulugeta. Magnitude of Cryptococcal Antigenemia among HIV Infected Patients at a Referral Hospital, Northwest Ethiopia. Ethiop J Health Sci.2018; 28(4): 369 .

doi: http:// dx. doi. org/10.4314/ejhs.v28i4.2

Received: December 20, 2017

Accepted: December 23, 2017

Published: July 1, 2018

Copyright: (C) 2018 Awoke Derbie, et al .

This is an open access article distributed under the terms of the Creative Commons Attribution License, which permits unrestricted use, distribution, and reproduction in any medium, provided the original author and source are credited. Funding: Nil

Competing Interests: The authors declare that this manuscript was approved by all authors in its form and that no competing interest exists.

Affiliation and Correspondence:

${ }^{1}$ Department of Medical Microbiology,

Immunology and Parasitology, College of Medicine and Health Sciences, Bahir Dar University, Bahir Dar, Ethiopia

${ }^{2}$ CDT-Africa, Addis Ababa University, Ethiopia

${ }^{3}$ Felege Hiowot Referral Hospital, Bahir Dar, Ethiopia

${ }^{4}$ Biotechnology Research Institute, Bahir Dar University, Ethiopia

${ }^{5}$ Department of Epidemiology and Biostatstics, College of Medicine and Health Sciences, Bahir Dar University, Bahir Dar, Ethiopia

*Email: awe.love2000@gmail.com

\section{ABSTRACT}

BACKGROUND: Cryptococcosis is one of the common opportunistic fungal infections among HIV infected patients living in Sub-Saharan Africa, including Ethiopia. The magnitude of the disease at Felege Hiwot Referral Hospital (FHRH) in particular and in Ethiopia at large is not well explored.

METHODS: $A$ retrospective document review and analysis was done on records of $137 \mathrm{HIV}$ infected patients who visited FHRH ART clinic from 1 Sep to 30 Dec 2016 and had registered data on their sex, age, CD4 count and cryptococcal antigen screening result. The cryptoccocal antigen (CrAg) detection was done by the IMMY CrAg ${ }^{\circledR}$ LFA (Cryptococcal Antigen Lateral Flow Assay) kit from patient serum as per the manufacturer's instruction. All data were entered, cleared, and analyzed using SPSS v20. Descriptive data analysis and cross tabulation were done to assess factors associated with cryptococcal antigenemia. Statistical significance was set at p-value less than or equal to 0.05 .

RESULTS: More than half of the participants, $54.7 \%$ (75/137), included in the study were females. The median age of the participants was 32.0 years (ranged: 8-52 years). The mean CD4 count was 51.8 with $S D$ of 26.3 (range 3-98). All the patients were $H I V$ stage IV. The proportion of positive cryptococal antigen from serum test was at $11.7 \%$ (95\% CI: 7.3-18.1\%). The IMMY CrAg ${ }^{\circledR} \mathrm{LFA}$ result was found statically associated with patient sex ( $p=0.045)$. However, it was not associated with patient age group and the CD4 count $(P>0.05)$

CONCLUSIONS: This study provided baseline data on the magnitude of cryptococcal antigenemia among HIV positive patients that is not touched before in the studied area. The results of the study showed that this opportunistic fungal infection is an important health concern among HIV patients. Further studies with sound design employing adequate sample size should be considered.

KEYWORDS; Cryptococcal antigenemia, IMMY CrAg ${ }^{\circledR} L F A$, Bahir Dar, Ethiopia 


\section{INTRODUCTION}

Increasing access to antiretroviral therapy (ART) has transformed the prognosis of HIV infected patients in resource-limited settings. However, treatment coverage remains relatively low, and HIV diagnosis occurs at a late stage. As a result, many patients continue to die of HIV-related opportunistic infections (OIs) in the weeks prior to, and months following initiation of $\operatorname{ART}(1,2)$. Cryptococcus neoformans, a causative agent of cryptococcosis remain a common cause of infectious morbidity and mortality, especially among HIV-positive patients living in SubSaharan Africa (3). Cryptococcal disease is one of the most important OIs, and a major contributor to this early mortality, accounting for between $13 \%$ and $44 \%$ of deaths among HIV infected people living in developing nations. In sub-Saharan Africa alone, there are more than 500,000 deaths each year due to cryptococcal meningitis (CM), which may exceed those attributed to tuberculosis (1).

Cryptococcus is a type of fungus that lives in soil, especially soil that is contaminated with large amounts of bird droppings. Some people inhale the spores from the environment and never get sick, but in people with weak immune systems, the fungus can cause an infection. The only way a person can get sick from this fungus is by directly inhaling the agent from the environment, making it no person to person transmission $(1,4)$.

Cryptococcal infection is associated with a range of illness. In some people, it causes a lung infection similar to tuberculosis, or it can cause no symptoms at all. The incubation period is not known, but it is thought that the infection can remain dormant in the body for many years. In immunosuppressed people, particularly HIVinfected once with CD4 counts less than 100, the infection can reactivate and spread throughout the body. When this happens, the infection usually presents as meningitis which is a common cause of death among HIV/AIDS patients (5).

The case fatality rate in patients with cryptococcal meningitis, the commonest presentation of HIV-related cryptococcal disease in adults, remains unacceptably high, particularly in sub-Saharan Africa, between 35\%-65\%. This compares with $10 \%-20 \%$ in most developed countries. The main reason for this is a delay in presentation with diagnosis only when meningitis is advanced and treatment is less effective, mainly as a result of limited access to lumbar puncture (LP) and rapid diagnostic assays (1).

There are three categories of methods that can be used to diagnose cryptococcal meningitis: India Ink microscopy, which can be used on cerebrospinal fluid (CSF); culture, which can be used on CSF or blood; and antigen detection. There are several methods to detect cryptococcal antigen in CSF or serum: latex agglutination (LA), enzyme immunoassay (EIA), and lateral flow assay (LFA) (6-8). A patient who tests positive for cryptococcal antigen can take oral fluconazole to help the body fight the early stage of the infection. This could prevent the infection from developing into meningitis (9).

In Ethiopia, where there is poor surveillance system, there are few studies conducted on the level of cryptococcal infection and its associated risk factors $(2,10-11)$. However, the data is missing in our study site where there are a number of HIV patients getting ART service in accordance with the national ART program. Some HIV patients in the study area get tested for cryptococcal infection during follow-up when there is suggestive clinical presentations. Thus, the aim of this study was to assess the prevalence of cryptococcal antigenemia and its associated factors among HIV infected patients at Felege Hiwot Referral Hospital (FHRH) setting.

\section{MATERIALS AND METHODS}

Study design, setting and data collection: A retrospective record review was done on records of 137 HIV infected patients who were attending ART monitoring at FHRH ART clinic. The hospital is located in Bahir Dar town, which is the capital of Amhara National Regional State located $565 \mathrm{~km}$ away from the capital Addis Ababa. The FHRH is a tertiary health care level hospital serving the population of Bahir Dar and remote areas of Northwest Ethiopia. The total population served by the hospital is about 12 million. In the

DOI: http://dx.doi.org/10.4314/ejhs.v28i4.2 
hospital, the ART clinic is operating under the National HIV Program of Ethiopia, under which patients are diagnosed for HIV and get ART treatment and follow-up services (CD4 count, liver and kidney function tests and hematologic evaluation) for free.

HIV positive patients, regardless of their ART status, whose CD4 count was less than 100 and had headache with signs suggestive of meningitis were screened for $\mathrm{CrAg}$ in the hospital (1). Taking this background, those HIV patients who visited the hospital's ART clinic from 1 Sep to 30 Dec 2016 and had registered data on their sex, age, CD4 count and cryptococcal antigen screening result were included for analysis. Patient records that missed one of these variables were excluded from the study. Data were retrieved directly from laboratory registration logbook using data extraction sheet on 1to 10 January 2017.

Cryptococcal antigen test: Although culture is the standard method for definitive diagnosis, detection of cryptococcal antigen in serum or cerebrospinal fluid is used for presumptive diagnosis. Cryptococcal antigen screening in peripheral blood is also recommended for HIVinfected persons with CD4 cell counts $<100 / \mu 1$ to reduce early deaths while receiving $\operatorname{ART}(6,12)$.

In the studied area, the cryptoccocal antigen (CrAg) detection was done by the IMMY CrAg ${ }^{\circledR}$ LFA (Cryptococcal Antigen Lateral Flow Assay) kit as per the manufacturer's instruction. The IMMY $\mathrm{CrAg}^{\circledR} \mathrm{LFA}$ is an immunochromatographic dipstick assay for the qualitative and semi-quantitative detection of cryptococcal antigen in serum, plasma, whole blood and cerebrospinal fluid (CSF). The $\mathrm{CrAg}^{\circledR}$ LFA is a prescription use laboratory assay which can aid in the diagnosis of cryptococcosis $(6,12)$. In this study, from those patients who came with evidence of cryptococcal meningitis, $40 \mu 1$ serum was used to detect the $\mathrm{CrAg}$. The serum and its one drop dilute were added into a test tube and the lateral flow device that is pre-coated with anti$\mathrm{CrAg}$ monoclonal antibodies and gold conjugated control antibodies was submerged with its white end. After ten minutes of incubation, the result was read and recorded. The antigen antibody complex forms a test line causing a visible line to form. The IMMY $\mathrm{CrAg}^{\circledR}$ LFA has sensitivity and specificity of $100 \%$ using serum (12-13). Patients found positive for $\mathrm{CrAg}$ were managed using fluconzole based antifungal treatment based on the recommended approach.

Statistical analysis: All data were entered, cleared, and analyzed using SPSS statistical software package, Version 22.0. Descriptive data analysis was used to visualize differences within data, and cross tabulation was done to assess factors associated with cryptococcal antigen. Differences were considered significant when $p$ value was less than or equal to 0.05 .

Ethical issues: Permission and ethical clearance were obtained from Amhara Regional Health Bureau Institutional Review Board (IRB) to utilize the data. As the data was collected retrospectively, no patient details were linked to the patient's identity and confidentiality was maintained.

\section{RESULTS}

In this study, we analyzed the records of $137 \mathrm{HIV}$ infected patients to assess their cryptococcal antigenemia. More than half of the participants, $75(54.7 \%)$, were females. The median age of the participants was 32.0 years (ranged: 8-52 years). The mean CD4 count was at 51.8 with standard deviation of 26.3 (range 3-98). The proportion of positive cryptococal antigenemia from serum test was at $11.7 \%$ (95\% CI: 7.3-18.1\%) (Table1). Further, based on the WHO classification system, all of the HIV positive participants in this study were stage IV. 
Table 1: Demographic and related clinical data of HIV patients screened for cryptococcal antigenemia at FHRH, Bahir Dar, 2016.

\begin{tabular}{llll}
\hline Variable & & Frequency & Percent \\
Sex & Male & 62 & 45.3 \\
& Female & 75 & 54.7 \\
Age group in years & Total & 137 & 100 \\
& $0-14$ & 3 & 2.2 \\
& $15-24$ & 11 & 8.0 \\
& $25-34$ & 69 & 50.4 \\
& $35-44$ & 38 & 27.7 \\
CD4 count/ $\boldsymbol{\mu l}$ & $>44$ & 16 & 11.7 \\
& $0-25$ & 25 & 18.2 \\
& $26-50$ & 46 & 33.6 \\
CrAg LFA result & $51-75$ & 36 & 26.3 \\
& $76-100$ & 30 & 21.9 \\
& Positive & 16 & 11.7 \\
\hline
\end{tabular}

We found that the IMMY $\mathrm{CrAg}^{\circledR}$ LFA result was statically associated with patient $\operatorname{sex}(p=0.045)$. However, it was not found associated with the patient age group and the CD4 count $(P>0.05)$ (Table).

Table 2: CrAg LFA test result of study subjects at FHRH, Bahir Dar, 2016.

\begin{tabular}{|c|c|c|c|c|c|}
\hline \multirow{2}{*}{\multicolumn{2}{|c|}{ Variable }} & \multicolumn{4}{|c|}{ *CrAg LFA result } \\
\hline & & No. Tested & Positive $\mathrm{n}(\%)$ & Negative n (\%) & Pvalue \\
\hline \multirow[t]{2}{*}{ Patient Sex } & Male & 62 & $11(17.7)$ & $51(82.3)$ & \\
\hline & Female & 75 & $5(6.7)$ & $70(93.3)$ & 0.045 \\
\hline \multirow{5}{*}{$\begin{array}{l}\text { Age groups in } \\
\text { years }\end{array}$} & $0-14$ & 3 & $1(33.3)$ & $2(66.7)$ & \\
\hline & $15-24$ & 11 & $0(0)$ & $11(100)$ & \\
\hline & $25-34$ & 69 & $7(10.1)$ & $62(89.9)$ & 0.276 \\
\hline & $35-44$ & 38 & 7 (18.4) & $31(81.6)$ & \\
\hline & $>44$ & 16 & $1(6.3)$ & $15(93.7)$ & \\
\hline \multirow[t]{4}{*}{ CD4 count } & $0-25$ & 25 & $3(12)$ & $22(88)$ & \\
\hline & $26-50$ & 46 & $7(15.2)$ & $39(84.8)$ & 0.793 \\
\hline & $51-75$ & 36 & $3(8.3)$ & 33 (91.7) & \\
\hline & $76-100$ & 30 & $3(10)$ & $27(90)$ & \\
\hline
\end{tabular}

*CrAg LFA: Cryptococcal antigen lateral flow assay test

\section{DISCUSSION}

Although the widespread availability of antiretroviral therapy (ART) in developed countries has helped reduce cryptococcal infections in these areas, it is still a major problem in developing countries, like Ethiopia, where access to healthcare is limited (9). Most of the patients with cryptococcal meningitis have serious disease and high fatality rate, and its clinical symptoms are not typical, misdiagnosis is common in early stage (4).

Each year, Cryptococcus is believed to cause more deaths than tuberculosis in Sub-Sahran Africa (14-15). Therefore, screening individuals with AIDS for serum cryptococcal antigen (CrAg), followed by treatment of $\mathrm{CrAg}$ positives 
with antifungals, may prevent cryptococcal meningitis $(2,6,15)$. In this study, most of the participants living with HIV were females (54.7\%). Similarly, in terims of their age category, the majority of HIV patients, $50.4 \%$ were in the 25-36 years group which implies that HIV still affects those individuals who are sexually active. Moreover, there is a similar report by Kharsany et al's study in which adolescent girls and young women aged 15-24 years had up to eight fold higher rates of HIV infection compared to their male peers. There remains a gap in women initiated HIV prevention technologies especially for women who are unable to negotiate the current HIV prevention options of abstinence, behavior change, condoms and medical male circumcision or early treatment initiation in their relationships (16).

In this study, we found that the overall prevalence of cryptococcal antigenemia was $11.7 \%$ among HIV infected patients with mean CD4 count at 51.8 cells $/ \mu 1$. According to Shiferaw et al's study, in Addis Ababa-Ethiopia, the magnitude of cryptococcal antigenemia was $11 \%$ among HIV infected patients with CD4 count lessthan 100 cells $/ \mu 1$ (10). Similarly, according to Bitew et al's. study in Addis Ababa, the prevalence of cryptococcal antigenemia among HIV positive patients was $8.5 \%$ (11). An overall prevalence of $9.9 \%$ of $C$. neoformans infection among HIV patients was also documented by Egbe et al. in Nigeria (17) which makes our finding almost comparable with previous studies done in Ethiopia and elsewhere in the world.

It is indicated that among immunosuppressed persons, particularly HIV-infected people with CD4 counts under 100, cryptococcal infection is important (16) and is one of the AIDS defining infections (3). Cryptococcal antigenemia was not found associated with patient age group and their CD4 count $(P>0.05)$ (Table 2$)$. On a study done in Nigeria, authors demonstrated that CD4 count less than $200 / \mu 1$ was significantly associated with cryptococcal antigenemia but age group and gender did not show association (17). The statistical difference on CD4 might be because of the small sample size we have employed in the present study.
In this study, we found that the mean CD4 count of the study participants was 51.8 per $1 \mu 1$ of blood (range 3-98). At the same time, all of them were stage IV. Considering the fact that in such patients with CD4 counts $<100$, screening them for CrAg and early ART initiation is the most important and cost-effective preventive strategy to reduce the incidence and high mortality associated with cryptococcal meningitis in HIV-infected patients (1). Since Amphotericin B is not available, the management protocol of $\mathrm{CrAg}$ positive HIV patients in FHRH was using fluconzole based antifungal treatment based on the WHO recommended approach (1) and an expert's opinion. The fluconzole based treatment was given in three phases: 1) induction phase (for two weeks, high dose-600mg BID), 2) consolidation phase (for up to eight weeks, 400mg BID) and 3) maintenance phase $(200 \mathrm{mg}$ daily for 3-6 months until the patients attains CD4 count of >200). Lumber puncture was also given every 24 hours for these patients until they got relief from the headache.

The results of our study should be applied with caution. Our findings are subject to at least two limitations: selection bias and quite limited sample size. At the same time, due to the retrospective nature of the study, it was not possible to show detailed clinical picture of HIV patients which might have a significant role to indicate the overall profile of the study participants. No data was found on the ART status and antifungal treatment outcome of patients with positive CrAg test. However, our study is the first of its kind in the studied area that provide baseline information about the magnitude of cryptococcosis among HIV positive patients for further large scale study. The results of this study also showed that this opportunistic fungal infection is an important health problem among HIV patients that needs the attention of physicians who are in charge of attending them.

In conclusion, althrough we have employed quite limited study participants' data, the reported prevalence of cryptococcal antigenemia calls stakeholders to expand CrAg screening service for individuals with HIV/AIDS, especially for those with CD4 count $<100 / \mu 1$. Additional prospective

DOI: http://dx.doi.org/10.4314/ejhs.v28i4.2 
study with adequate sample size is needed to determine the exact magnitude of the disease and to explore its determinants.

\section{ACKNOWLEDGEMENTS}

We express our deep appreciation to FHRH laboratory staffs for their support during data collection. Our great appreciation is also goes to Bahir Dar University for provision of facilities to exercise research.

\section{REFERENCES}

1. World Health Organization (WHO). Rapid advice: diagnosis, prevention and management of cryptococcal disease in HIV-infected adults, adolescents and children. 2011. $<$ http://apps.who.int/iris/bitstream/10665/44786/ 1/9789241502979 eng.pdfs

2. Beyene $\mathrm{T}$, Woldeamauel $\mathrm{Y}$, Asrat $\mathrm{D}$ et al. Comparison of Cryptococcal Antigenemia between Antiretroviral Nai"ve and Antiretroviral Experienced HIV Positive Patients at Two Hospitals in Ethiopia. PLoS ONE 8 (10): e75585.

3. Lungran $\mathrm{P}$, Vijaya $\mathrm{D}$, Shashi $\mathrm{S}$, et al. Cryptococcosis: Its Prevalence and Clinical Presentation among Hiv Positive and Negative Patients in Rims, Manipur. IOSR Journal of Dental and Medical Sciences. 2014;13(7):38.

4. Wang H, Yuan X, Zhang L. Latex agglutination: Diagnose the early cryptococcus neoformans test of capsular polysaccharide antigen. Pak. J. Pharm. Sci., 2015; 28(1):307-311.

5. Aberg A, Powderly G. Cryptococcosis and HIV. Available at $<$ http://hivinsite.ucsf.edu/InSite? page $=\mathrm{kb}-05-02-$ 05>. Accessed on 28 July 2017

6. Boulware R, Rolfes A, Rajasingham R, et al. Multisite Validation of Cryptococcal Antigen Lateral Flow Assay and Quantification by Laser Thermal Contrast. Emerging Infectious Diseases 2014; 1. <www.cdc.gov/eid >

7. Saha C, Bhowmik M, Xess I, Padma V, Biswas A. Detection of Cryptococcus by conventional, serological and molecular methods. Journal of Medical Microbiology. 2009; 58: 1098-1105.
8. Subramanian S, Mathai D. Clinical manifestations and management of cryptococcal infection. J Postgrad Med. 2005; 51: Suppl 1: 21-6.

9. Centers for Disease Control and Prevention (CDC), National Center for Emerging and Zoonotic Infectious Diseases Division of Foodborne, Waterborne, and Environmental Diseases. Cryptococcal meningitis: a deadly fungal disease among people living with HIV/AIDS. http://www.cdc.gov/fungal/.

10. Alemu AS, Kempker RR, Tenna A, et al. High Prevalence of Cryptococcal Antigenemia among HIV-infected Patients Receiving Antiretroviral Therapy in Ethiopia. PLoS ONE 2013; 8(3): e58377. doi:10.1371/journal.pone.0058377

11. Bitew A, Hassen M, Getachew T, Fentaw S. Prevalence of Crytpococcal Infection in Patients Clinically Diagnosed to Have Meningitis in Ethiopia. Clinical Medicine Research. 2016;5 (4):73-76.

12. The IMMY CrAg LFA (Cryptococcal Antigen Lateral Flow Assay). Available at: http://www.immy.com/products/lateral-flowassays/crag-lfa/. Accessed on 30 Oct 2016.

13. Kozel R, Bauman K. CrAg Lateral Flow Assay for Cryptococcosis. Expert Opin Med Diagn. 2012; 6(3): doi:10.1517/17530059.2012.681300.

14. Warkentien T, Crum-Cianflone F. An Update on Cryptococcosis Among HIV-Infected Persons. Int J STD AIDS. 2010; 21(10): 679.

15. CDC: Cryptococcal Screening. A New Strategy for saving lives among people with HIV/AIDS. $<$ https://www.cdc.gov/fungal/pdf/crypto-screenstrategy-508c.pdf>.

16. Kharsany B and Karim A. HIV Infection and AIDS in Sub-Saharan Africa: Current Status, Challenges and Opportunities. The Open AIDS Journal, 2016; 10:34-48.

17. Egbe A, Omoregie R, Alex-Ighodalo O. Cryptococcus neoformans infection among human immunodeficiency virus patients on highly active antiretroviral therapy in Benin City, Nigeria. N Z J Med Lab Sci. 2015;69: 2123.

DOI: http://dx.doi.org/10.4314/ejhs.v28i4.2 